\title{
Do intermediate mass black holes exist?
}

\author{
Martin J. Ward ${ }^{1,2}$ \\ ${ }^{1}$ Department of Physics and Astronomy, University of Leicester, Leicester, LE1 7RH, UK \\ ${ }^{2}$ Present Address: Dept. of Physics, University of Durham, Durham, DH1 3LE, UK
}

\begin{abstract}
I review the evidence in favour of the existence of intermediate mass black holes (IMBH) in a sub-set of the class of ultraluminous X-ray source (ULX). Their X-ray properties and information on their local environments is beginning to point towards the presence of IMBHs in some, and perhaps the most luminous, ULX.
\end{abstract}

\section{Introduction}

First I define what I mean by intermediate mass black holes (IMBH). The term applies to the mass range in between the highest mass black hole that can be formed in a galactic binary system under the standard assumptions i.e. $\sim 20 \mathrm{M}_{\odot}$ (see Fryer \& Kalogera 2001), and the lowest mass black hole located at the dynamical centre of a galaxy - the record currently is for NGC4395, for which Fillippenko \& Ho (2003) argue that the mass lies in the range $\sim 104$ to $105 \mathrm{M}_{\odot}$. There are thus roughly three orders of magnitude between estimates of the highest mass galactic black hole, and the lowest mass AGN black hole. Potentially good candidates to populate the mid-range of $\sim 1000$ 's $\mathrm{M}_{\odot}$ would be globular clusters, however the evidence for IMBH in these systems is curently ambiguous (Gerssen et al. 2003).

In this review I will describe the evidence for the presence of IMBHs in some ULXs. An ultraluminous X-ray source (ULX) has $L_{\mathrm{X}}>10^{39} \mathrm{erg} \mathrm{s}^{-1}$. The name ULX is used because typical black-hole binaries known in our Galaxy (excluding the more exotic micro-quasars) are about an order of magnitude less luminous, and because it is assumed that ULXs are not a part of the same X-ray luminosity distribution as the much more luminous AGN. We should bear in mind that the luminosity threshold for ULXs of $10^{39}$ erg $\mathrm{s}^{-1}$ is an arbitrary one. However, if one assumes a specific model, i.e. symmetric accretion onto a $1.4 M_{\odot}$ compact object such as a neutron star, then this luminosity implies either a substantially super-Eddington accretion rate, or that the compact object is a black hole of many solar masses. We believe that ULXs are some type of binary system, and not SNRs, principally because when monitored the majority of them exhibit $\mathrm{X}$-ray variability, although as yet none has a confirmed periodicity. However, because of the crude single parameter definition of luminosity, a few of the brightest supernova remnants (SNR) may be included in any given sample of ULX. This small sub-set of ULX is not relevant to our discussion of evidence for IMBHs.

\section{The Role of Geometry}

The need to invoke IMBHs as an explanation for ULXs, based on the Eddington limit and their luminosities, may be removed if their X-ray emission is significantly beamed.

There are two classes of models that seek to explain ULXs in terms of anisotropic emission. In the first case a link has been suggested between ULXs and galactic superluminal sources (often referred to as galactic micro-quasars - GMQs) such as GRS1915+105, (see 
Körding et al. 2002). The X-ray spectral properties of some ULXs are similar to the GMQs, although there are exceptions.

The second class of anisotropic model invokes funnelled (not intrinsic) collimation via a thick accretion disc (King et al. 2001). They associate ULXs with close-separation X-ray binaries undergoing mass transfer on a thermal time-scale. Super-Eddington accretion can proceed as the donor overflows its Roche lobe. One constraint of the model is that the required donor star must be of early type, to avoid quenching of the X-rays in the resulting common envelope. The model naturally explains why ULXs are common in active starforming regions. This model has the advantage that the mass range required for the compact object is reduced by a factor of a few, and significantly it is a natural explanation based on established phenomena in known classes of object. However, difficulties remain to account for the most luminous ULXs.

\section{What can the X-ray properties of ULX tell us?}

Given the wealth of information derived from X-ray studies of AGN, one might hope for similar insights into the nature of ULXs by examination of their X-ray properties. As will be seen, this hope is borne out to some degree, but the situation is far from clear.

\subsection{The X-ray spectra of $U L X s$}

A few years ago it was realised that the X-ray spectra of many ULX systems could be modelled by fitting black-body emission from an optically thick standard accretion disc around a BH (Makishima et al. 2000). Since the integrated spectrum is the result of adding together regions of different temperature (producing corresponding thermal 'colours'), this fit is referred to as the multicolour disc (MCD) model. However, there are several problems with this model. The temperature of material at the inner accretion radius implied using $\mathrm{MCD}$ models is generally too high for the $\mathrm{BH}$ mass. One escape route for the MCD model is to postulate that the $\mathrm{BH}$ is spinning, and hence the inner radius is closer to the $\mathrm{BH}$ compared with a non-rotating $\mathrm{BH}$ of the same mass.

In addition, it was observed that not all X-ray spectra of ULXs are well fitted using the MCD model, counter examples being the ULXs in Holmberg II (Miyaji et al. 2001), IC342 (Kubota et al. 2001) and NGC 3628 (Strickland et al. 2001), which require the presence of a hard spectral component with photon index 1-2. The soft component may originate from an optically thick accretion disc, with the hard component arising from Comptonization of soft photons by hot electrons in an extended medium (the corona) located above and below the accretion disc.

Following XMM-Newton spectral studies of ULXs of better quality than available previously, our view has been modified. It now appears that there are many exceptions to ULX spectra fitted by hot disc MCD components, with or without the need for an additional power-law. Noteably, many of the ULXs at the high end of their luminosity distribution ie. $L_{\mathrm{X}}>10^{40} \mathrm{erg} \mathrm{s}^{-1}$ have X-ray spectra well fitted by inner disc temperatures in the range 100-300 eV. It could be, as pointed out by Miller \& Colbert (2004), that above some luminosity threshold the IMBH explanation becomes favoured over versions of the models of microquasar or the funnelled emission from high mass X-ray binaries (HMXB) mentioned earlier, as these have problems producing such high luminosities.

\subsection{X-ray Variability}

X-ray variability is a hallmark of ULXs. In some cases this is spectacular as for the very luminous ULX in M82 which varied by a factor of 7 between two Chandra observations taken 3 months apart. Also, large amplitude variablity over medium/long timescales are 
seen in ULXs in the Antennae, M81 and M51. In a large statistical study Colbert \& Ptak (2002) claimed $>50 \%$ variability in more than half their sample of ULX. Whilst these data support the binary nature of ULX they do not provide evidence for IMBHs in them. For this we must look for short term coherent variations.

There are several claims of possible periodic behaviour in ULXs. For the source Circinus CGX-1, Bauer et al (2001) claim periodic variations of 7.5 hours, and argue against it being a foreground galactic binary because of the absence of an optical counterpart to $m_{\mathrm{v}}>25.3$ magnitudes, and on general statistical grounds. The other possibly periodic ULX is in the galaxy IC342 (Sugiho et al. 2001) and has a suggested period of 31 hours or 41 hours, which is consistent with the orbital period of a semidetached binary with a $\mathrm{BH}$ and a main-sequence star of several tens of solar masses. Recently Terashima \& Wilson (2003) reported a ULX in M51 with a > 50\% variation separated by about two hours. Based on the present data none of these observations can be taken as firm evidence for periodicity in a ULX, but clearly continued monitoring over a range of time scales is essential.

Finally, I mention the shortest timescales of variability associated with ULX. So far data is very limited. The brighest source in M82, also one of the most luminous ULXs known, was found to exhibit quasi-periodic oscillations (QPO) by Strohmayer \& Mushotzky (2003) using XMM. This could be taken as some support for the IMBH hypothesis in this case, not actually based on the QPO timescale but rather on the fact that we see it at all. This argues against significant beaming, which would dilute the disc component in which the QPO originates. Without beaming the Eddington constraint suggests a $\mathrm{BH}$ mass of not less than $\sim 300 \mathrm{M}_{\odot}$. The other intriguing result on short term variability is reported by Cropper et al. (2004), who studied the bright ULX, NGC4559$\mathrm{X} 7\left(L_{\mathrm{X}}>10^{40} \mathrm{erg} \mathrm{s}^{-1}\right)$ and found a break in its power-density spectrum at $28 \mathrm{mHz}$. This, coupled with an X-ray spectral fit to a black body component of $120 \mathrm{eV}$, favours an IMBH, but with very model dependent estimates, giving $\sim 40$ to $800 \mathrm{M}_{\odot}$.

\section{Location of ULXs: host galaxy, local environment and counterparts}

ULXs are found across all Hubble types of galaxies. However for the purpose of this review I will ignore elliptical galaxies, whilst noting that some ULXs in these may harbour IMBHs in their associated globular clusters. In the case of spiral and irregular galaxies a general link between ULX and starformation is apparent, eg. M82, the Antennae, NGC3256 and the Cartwheel, which supports the association of many ULX with HMXBs (Gao et al. 2003).

Turning to the local environments of ULX, much effort has recently focussed on searching for X-ray driven ionised nebulae. Nebulae have been detected around several ULX eg. IC 342 (Roberts et al. 2003) and NGC1313-X2 (Zampieri et al. 2004), although it is unclear whether these nebulae are shock or photionised. In both cases the energetics imply either a hypernova or multi-supernovae, and X-ray variability strongly suggests that these ULXs are binary systems located within the nebula. A particularly interesting case of a nebula surrounding a ULX is Holmberg II (Pakull \& Minioni 2003; Kaaret et al. 2004). Using Chandra and HST data, Kaaret et al. (2004) determined the energetics of the nebulae based on the high excitation optical emission line of HeII. Their data suggests that the emission from the ULX is not significantly beamed, and hence the black hole would have a mass in the range $\sim 25$ to $40 \mathrm{M}_{\odot}$.

Next I come to claims of compact counterparts to ULX. The difficulty caused by confusion of sources in this work is clearly seen by inspection of high spatial resolution optical images of two ULXs (Goad et al. 2002, Zampieri et al. 2004). Indeed, even when 
optical/UV spectra are available for the faint counterpart (Liu et al. 2004), information on the mass of the primary component is of limited value. However, a hope for the future is that eventually a period can be determined in a ULX, leading to a reliable mass estimate independent of those based on X-ray spectra and luminosity. For completeness I note that radio observations may prove useful in the future. For example, the very luminous ULX in M82 is spatially consistent with a bright radio transient that was detected in the 1980's, but which has not been seen since. The link between ULX and galactic microquasars is strengthened by the association of radio emission with the ULX in NGC5408 (Kaaret et al. 2003). But, to explain the strength of the radio emission the jet must lie close to our line of sight, making it a micro-blazar.

\section{Conclusions}

The evidence for IMBHs in some ULX is intriguing but not yet conclusive. In seems plausible that the high luminosity ULXs with $\left(L_{\mathrm{X}}>10^{40} \mathrm{erg} \mathrm{s}^{-1}\right)$ are the best candidates for IMBHs. Their formation and feeding remain major problems, but this may be incorporated with that for supermasssive BHs, if these ULX are the cores of captured dwarf companion galaxies. For example, the ULX in M81-X9 is possibly associated with Holmberg IX, a dwarf companion galaxy to M81, Wang (2002).

\section{References}

Bauer, F. E., et al. 2001, AJ, 122, 182

Bicknell, G. V., Dopita, M. A., Tsvetanov, Z. I., \& Sutherland, R. S. 1998, ApJ, 495, 68

Colbert, E. J. M., \& Ptak, A. F. 2002, ApJS, 143, 25

Cropper, M., Soria, R., Mushotzky, R. F., Wu, K., Markwardt, C. B., \& Pakull, M. 2004, MNRAS, 349, 39

Filippenko, A. V., \& Ho, L. C. 2003, ApJ, 588, L13

Fryer, C. L., \& Kalogera, V. 2001, ApJ, 554, 548

Gao, Y., Wang, Q. D., Appleton, P. N., \& Lucas, R. A. 2003, ApJ, 596, L171

Gerssen, J., van der Marel, R. P., Gebhardt, K., Guhathakurta, P., Peterson, R. C., \& Pryor, C. 2003, AJ, 125, 376

Goad, M. R., Roberts, T. P., Knigge, C., \& Lira, P. 2002, MNRAS, 335, L67

Kaaret, P., Ward, M. J., \& Zezas, A. 2004, MNRAS, 351, L83

Kaaret, P., Corbol, S., Prestwich, A. H., \& Zezas, A. 2003, Science 299, 365

King, A. R., Davies, M. B., Ward, M. J., Fabbiano, G., \& Elvis, M. 2001, ApJ, 552, L109

Körding, E., Falcke, H., \& Markoff, S. 2002, A\&A, 382, L13

Kubota, A., et al. 2001, ApJ, 547, L119

Liu, J-F, Bregman, J. N., \& Seitzer, P. 2004, ApJ, 602, 249

Makishima, K., et al. 2000, ApJ, 535, 632

Miller, C. M., \& Colbert, E. J. M. 2004, Int. J. Mod. Phys., D13, 1

Miyaji, T., Lehmann, I., \& Hasinger, G. 2001, AJ, 121, 3041

Pakull, M. W., \& Mirioni, L. 2003, RMxAA, 15, 197

Roberts, T. P., Goad, M. R., Ward, M. J., \& Warwick, R. S. 2003, MNRAS, 342, 709

Strickand, D. K., Colbert, E. J. M., Heckman, T. M., Weaver, K. A., Dahlem, M., \& Stevens, I. R. 2001, ApJ, 560, 707

Strohmayer, T. E, \& Mushotzky, R. F. 2003, ApJ, 586, L61

Sugiho, M., et al. 2001, ApJ, 561, L73

Terashima, Y, \& Wilson, A. S. 2003, ApJ, 583, 145

Wang, Q. D. 2002, MNRAS, 332, 762

Zampieri, L., et al. 2004, ApJ, 603, 523 